\title{
EDITORIAL
}

\section{Idiopathic pulmonary fibrosis: treatment options in pursuit of evidence-based approaches}

\author{
G. Raghu
}

$\mathbf{T}$ he long-standing need to determine an effective medical treatment regimen with clinically significant and relevant end-points to improve outcome for patients with idiopathic pulmonary fibrosis (IPF) is being pursued by investigators in several phase I, II and III clinical trials. Patients confronted with IPF are understandably frustrated and vulnerable to hearsay and are desperate to try any medication in the hope of easing their suffering and increasing the likelihood of enhanced survival. This is particularly so when such medicines are available for clinical use either by prescription and/or over the counter. Caring physicians, also frustrated with the lack of evidence for an effective IPF treatment regimen, may find themselves reaching out for anecdotal reports and reacting to signals generated by post hoc exploratory and subgroup analyses derived from clinical studies, wanting to try some treatment regimen for their patients. In this regard, the joint American Thoracic Society/ European Respiratory Society international consensus statement on IPF provided a guideline pending documentation of an effective treatment regimen [1]. Acknowledging the limitations of the then-existing skeletal data, low-dose prednisone plus azathioprine or cyclophosphamide was suggested as a treatment regimen for patients with IPF, and an apparent "standard of care" for treatment of IPF with prednisone and azathioprine has evolved worldwide. The statement contained the disclaimer that its recommendation was based on the consensus of opinion of the expert panel, and the approach needs to be revisited as there was no grade-A evidence to support the treatment guidelines.

The widely acknowledged notion that the currently available treatment regimen is ineffective is largely based on individual physicians' experience. While this may be appropriate for treatment with lone corticosteroid therapy [2], colchicine [3, 4], cyclophosphamide [5,6] and d-penicillamine [7], care should be taken in generalising the notion to treatment with all currently available drugs. For instance, several years ago, combined low-dose prednisone plus azathioprine, compared with low-dose prednisone alone, did produce significant positive signals in a prospective, double-blind, randomised, placebo-controlled study in patients with newly diagnosed IPF [8]. Since no studies to date have compared the therapeutic

University of Washington, Chest Clinic, Interstitial Lung Disease/Sarcoidosis/Pulmonary Fibrosis Program, Lung Transplant Program, University of Washington Medical Center, Seattle, WA, USA.

CORRESPONDENCE: G. Raghu: Box 356166, 1959 Pacific Avenue, Seattle, WA 98195, USA. Fax: 1 2065982105. E-mail: graghu@u.washington.edu effects of combined prednisone plus azathioprine, or any other agents with a "true placebo" arm in newly diagnosed IPF, it is not known whether combined treatment with prednisone and azathioprine or another available treatment regimen is truly ineffective for IPF. Recently, the European Idiopathic Pulmonary Fibrosis International Group Exploring N-acetylcysteing (NAC) I Annual (IFIGENIA) trial documented a better preservation of forced vital capacity (FVC) and diffusing capacity of the lung for carbon monoxide $(D \mathrm{~L}, \mathrm{CO})$ in patients treated with low-dose prednisone, azathioprine and NAC compared with low-dose prednisone and azathioprine [9]. While this is indeed encouraging [10], the study raises the need to consider the potential of treatment with NAC alone. Given the known long-term side-effects associated with prednisone and azathioprine, there are appropriate reservations about using these drugs. The biological rationale for the use of NAC as an antioxidant and an anti-fibrotic agent in IPF has recently been reviewed [11]. Thus, it is possible that treatment with NAC alone may demonstrate stabilisation or decrease the rate of disease progression in IPF. Since NAC is available over the counter as an antioxidant, patients may be tempted to add NAC to their list of multivitamins, antioxidants and supplements with the hope of staying "healthy". Thus, the current standard of care for patients with IPF seems to be evolving into a status of subjective perception based on signals from studies rather than grade-A evidence. Physicians and patients need to be reminded, and taught, that only a well-designed, doubleblind, randomised, placebo-controlled, prospective clinical trial using NAC alone or any other active agent (with controls receiving only the placebo and disallowed from using concurrent medications that have potential treatment benefit) can provide the much-needed scientific evidence for treatment recommendations for IPF.

Several newer agents have the biological plausibility to block the inflammatory and fibrotic effects of specific cytokines and chemokines implicated in the pathogenesis of IPF [12]. These include interferon (IFN)- $\gamma$, endothelin-1 receptor antagonists and tyrosine kinase inhibitors. Their availability for clinical use increases the likelihood of the affected patient confronted with severely decreased life expectancy and the caring physician trying one or several of these agents. If physicians do this in the context of clinical observations in well-informed patients, useful clinical information gathered may enhance clinical understanding and aid better design of further studies. For instance, observations with the use of IFN- $\gamma$ in patients with advanced IPF do not suggest a therapeutic role in this subgroup of patients $[13,14]$. 
In the current issue of the European Respiratory Journal, ANTONIOU et al. [15] report their observation in a cohort of patients with IPF treated with IFN- $\gamma-1 b$. The investigators prospectively randomised a selected subgroup of patients with IPF (mild-to-moderate functional impairment, based on FVC $55 \%$ predicted and $D \mathrm{~L}, \mathrm{CO} 35 \%$ pred) to receive IFN- $\gamma-1 \mathrm{~b}$ or colchicine and followed their clinical course. It is apparent that the investigators had initially intended to study the antifibrotic effects of IFN- $\gamma-1 b$ with biomarkers, but they did not seem to get sufficient samples from patients to pursue this avenue. Nevertheless, the observed difference in survival favouring the treatment with IFN- $\gamma-1 b$ over colchicine is of interest. However, any enthusiasm is curbed by the poor design of the study.

One major flaw is the lack of pre-specified end-points in the protocol; as a consequence, the results need to be interpreted with caution, as emphasised recently [16]. The apparent survival benefit observed in the selected patient population in this small study needs further clarification in well-designed studies with appropriate sample sizes. An ongoing large multicentre clinical trial (the INSPIRE trial) is in fact targeted to determine whether there is a survival benefit with IFN- $\gamma$ treatment in this subgroup of patients with mild-to-moderate disease, as suggested by post hoc analyses in the first large phase III clinical trial [17]. In this regard, this large trial failed to confirm the positive findings reported initially in a small group of patients with IPF [18]. Until the results of the INSPIRE trial become available, it will remain unknown whether IFN- $\gamma$ is beneficial for IPF. Thus, provocative observations in small studies must not be immediately translated to altered standards of care for complex medical problems, such as IPF, until confirmed in well-designed, phase III studies.

Hopefully, the results of well-designed clinical trials will provide the necessary evidence to support clinical decisions for treatment. It is also hoped that some of the ongoing and planned multicentre clinical trials with other new agents (imitamib, zilueton, bosentan, pirfenidone, etc.) will aid progress towards improved outcomes for patients with IPF. However, response to any treatment regimen in an individual patient can only be predicted with reasonable accuracy by pharmacogenomic studies. The results of such studies are likely in future to provide better means of choosing between particular treatment regimens based on whether an individual is predisposed to respond.

During the past few years, clinicians, patients, investigators, funding agencies and pharmaceutical companies have joined forces to investigate new medications for IPF, and our knowledge of the clinical course of IPF in the context of prospective trials has been enhanced. While our understanding of the cellular and molecular mechanisms of IPF has evolved over the past few years, newer treatment strategies to modulate the fibrotic response in the lung are being investigated in clinical studies. Although the new concepts in the pathogenesis of IPF have been taken into account in the choice of agents in recent and ongoing clinical trials, the results of the studies using several of these novel agents (beta and gamma interferons [17, 19], pirfenidone [20], etanercept [21], and endothelin-1 receptor antagonist [22]) have not demonstrated significant differences between groups in the chosen primary end-points. Nevertheless, important hypotheses generated in subgroup analyses are already being tested in new clinical trials. These include awareness of the rapid decline of respiratory status preceding death in otherwise stable patients [23], remarkable stability in patients with IPF not receiving the active agent [24], importance of acute exacerbation of IPF [20], and the very low mortality during the first year of follow-up in patients with well-established IPF enrolled in these clinical trials $[9,19,20]$. In fact, this new awareness of the lack of significant progression in the placebo group observed over a period of 48-72 weeks in clinical trials using IFN- $\gamma[17,24]$, and in the IFIGENIA study [9], is somewhat reassuring. The feasibility of conducting clinical trials with a true placebo arm, and the potential ethical concerns about studying patients without any treatment appear to be less of an issue, based on current evidence.

While the current "standard of care" for IPF seems to have stemmed from the international consensus statement [1], the original clinical trial demonstrating a marginal survival benefit for patients with new-onset IPF following the initiation of combined prednisone and azathioprine as upfront treatment in an age-adjusted analysis needs to be tested in a large multicentre clinical trial [8]. The call for such a trial, made over 15 yrs ago, remains to be answered; in the interim, physicians have been forced to resolve this with their own individual judgement regarding the decision to treat patients with azathioprine. Thus, the standard of care with the use of prednisone and azthioprine has been variable and confounded with anecdotal reports and recommendations to use other immunosuppressive medications. Since the results from recent clinical trials using novel agents have been disappointing to date, further chaos in the standard of care is evident as clinicians yield to the temptation to try some of the new agents based on their own perception of the results disclosed.

It is therefore imperative for clinical investigators to clarify the current standard of care by providing new evidence. In this regard, the results from the IFIGENIA trial have awoken new interest in the unpopular regimen of low-dose prednisone combined with azathioprine and NAC. Given the new awareness of relative stability in patients with IPF followed prospectively in recent clinical studies, the possibility of clinical trials with a true placebo arm is now realistic. Thus, the need to improve the current chaotic and poor standard of care can effectively be met by comparing results with groups that include a true placebo arm. Such a well-designed clinical trial will separate myths from facts and provide evidence for a standard of care that can only be better than that being followed currently. Fortunately, several clinical trials are currently under way and more are being planned. While physicians and patients should continue to be made aware of all the clinical trials available to them, until the results of these studies become available, clinicians must provide the best supportive care for all patients with IPF and lung transplant must be considered in a timely manner for eligible patients. Physicians should monitor the clinical course of IPF regularly and promptly undertake appropriate diagnostic and therapeutic intervention for complications known to occur in patients with IPF. 
In essence, clinicians and patients must interpret new data on the treatment of idiopathic pulmonary fibrosis with caution [16]. This is especially necessary when interpreting results from studies with small patient numbers. Patients should be made fully aware of these concerns and be encouraged to participate in any well-designed clinical trials available to them. For patients who are unwilling, ineligible or unable to be enrolled in available clinical trials, a conservative follow-up with no "specific medications" other than oxygen supplementation and the best supportive care, based on clinical and physiological needs and close monitoring, is an appropriate option that should be discussed with the patient until an effective and safe treatment regimen is determined through clinical trials for idiopathic pulmonary fibrosis. The absolute need for a new and better standard of care for idiopathic pulmonary fibrosis patients will hopefully be met with evidence in the very near future.

\section{ACKNOWLEDGEMENTS}

I am deeply indebted to the community and academic clinicians and to all their patients who have allowed me to provide care for them. Without them I would not have gained experience in the management of interstitial lung diseases to share with the community. Assistance from C. Spada, J. Hayes and A. Snydsman, as well as L. Baumgart, J. Moniz and the staff of the clinic and pulmonary diagnostic services of the University of Washington Medical Center, is greatly appreciated.

\section{REFERENCES}

1 American Thoracic Society (ATS), the European Respiratory Society (ERS). Idiopathic pulmonary fibrosis: diagnosis and treatment. International consensus statement. Am J Respir Crit Care Med 2000; 161: 646-664.

2 Turner-Warwick M, Burrows B, Johnson A. Cryptogenic fibrosing alveolitis: response to corticosteroid treatment and its effect on survival. Thorax 1980; 35: 593-599.

3 Douglas WW, Ryu JH, Schroeder DR. Idiopathic pulmonary fibrosis: impact of oxygen and colchicine, prednisone, or no therapy on survival. Am J Respir Crit Care Med 2000; 161: 1172-1178.

4 Douglas WW, Ryu JH, Swensen SJ, et al. Colchicine versus prednisone in the treatment of idiopathic pulmonary fibrosis. A randomized prospective study. Members of the lung study group. Am J Respir Crit Care Med 1998; 158: 220-225.

5 Johnson MA, Kwan S, Snell NJ, Nunn AJ, Darbyshire JH, Turner-Warwick M. Randomised controlled trial comparing prednisolone alone with cyclophosphamide and low dose prednisolone in combination in cryptogenic fibrosing alveolitis. Thorax 1989; 44: 280-288.

6 Collard HR, Ryu JH, Douglas WW, et al. Combined corticosteroid and cyclophosphamide therapy does not alter survival in idiopathic pulmonary fibrosis. Chest 2004; 125: 2169-2174.

7 Selman M, Carrillo G, Salas J, et al. Colchicine, Dpenicillamine, and prednisone in the treatment of idiopathic pulmonary fibrosis: a controlled clinical trial. Chest 1998; 114: 507-512.
8 Raghu G, Depaso WJ, Cain K, et al. Azathioprine combined with prednisone in the treatment of idiopathic pulmonary fibrosis: a prospective double-blind, randomized, placebocontrolled clinical trial. Am Rev Respir Dis 1991; 144: 291-296.

9 Demedts M, Behr J, Buhl R, et al. High-dose acetylcysteine in idiopathic pulmonary fibrosis. $N$ Engl J Med 2005; 353: 2229-2242.

10 Wells AU. Antioxidant therapy in idiopathic pulmonary fibrosis: hope is kindled. Eur Respir J 2006; 27: 664-666.

11 Hunninghake GW. Antioxidant therapy for idiopathic pulmonary fibrosis. N Engl J Med 2005; 353: 2285-2287.

12 Selman M. Plunging into the chaos of the cytokine/ chemokine cocktail in pulmonary fibrosis: how many and how important are they? Am J Respir Crit Care Med 2003; 168: 730-731.

13 Kalra S, Utz JP, Ryu JH. Interferon gamma-1b therapy for advanced idiopathic pulmonary fibrosis. Mayo Clin Proc 2003; 78: 1082-1087.

14 Raghu G, Spada C, Otaki Y, Hayes JM. Interferon-gamma in the treatment of advanced idiopathic pulmonary fibrosis and fibrotic nonspecific interstitial pneumonia (NSIP-F): prospective, preliminary observation in one center. Chest 2001; 120: 185S.

15 Antoniou KM, Nicholson AG, Dimadi M, et al. Long-term clinical effects of interferon gamma-1b and colchicine in idiopathic pulmonary fibrosis. Eur Respir J 2006; 28: 496-504.

16 Johnson WC, Raghu G. Clinical trials in idiopathic pulmonary fibrosis: a word of caution concerning choice of outcome measures. Eur Respir J 2005; 26: 755-758.

17 Raghu G, Brown KK, Bradford WZ, et al. A placebocontrolled trial of interferon gamma- $1 b$ in patients with idiopathic pulmonary fibrosis. N Engl J Med 2004; 350: 125-133.

18 Ziesche R, Hofbauer E, Wittman K, Petkov V, Block LH. A preliminary study of long-term treatment with interferon gamma-1b and low-dose prednisolone in patients with idiopathic pulmonary fibrosis. N Engl J Med 1999; 341: 1264-1269.

19 Raghu G, Bozic CR, Brown $K$, et al. Feasibility of a trial of interferon beta-1A (IFN-beta-1A) in the treatment of idiopathic pulmonary fibrosis (IPF). Presented at the International Conference of the American Thoracic Society, 2001. Am J Respir Crit Care Med 2001; 163: A707.

20 Azuma A, Nukiwa T, Tsuboi E, et al. Double-blind, placebo-controlled trial of pirfenidone in patients with idiopathic pulmonary fibrosis. Am J Respir Crit Care Med 2005; 171: 1040-1047.

21 Raghu G, Lasky JA, Costabel U, et al. A randomized placebo controlled trial assessing the efficacy and safety of etanercept in patients with idiopathic pulmonary fibrosis (IPF). Chest 2005; 128: 496S.

22 King TE, Behr J, Brown K, DuBois R, Raghu G. Results of Build I Trial. Presented at the International Conference of the American Thoracic Society, 2006. Proceedings of the American Thoracic Society (PATS) 2006; 3: A524.

23 Martinez F, Safrin S, Wyecker D, et al. The clinical course of patients with idiopathic pulmonary fibrosis. Ann Intern Med 2005; 142: 963-967.

24 King TE Jr, Safrin S, Starko KM, et al. Analyses of efficacy end points in a controlled trial of interferon- $\gamma 1 \mathrm{~b}$ for idiopathic pulmonary fibrosis. Chest 2005; 127: 171-177. 